\title{
Velocidade de leitura e desempenho escolar na última série do ensino fundamental
}

\author{
Reading rate and academic performance in \\ the last grade of elementary school
}

\author{
Eliana Matiko KOMENO' \\ Clara Regina Brandão de ÁVILA² \\ Isa de Pádua CINTRA ${ }^{3}$ \\ Teresa Helena SCHOEN ${ }^{4}$
}

\begin{abstract}
Resumo
A velocidade de leitura, calculada pelo número de palavras lidas por minuto, é um dos aspectos observados na fluência dessa habilidade. Esta pesquisa caracterizou a velocidade de leitura no último ano do ensino fundamental, segundo o texto e o desempenho acadêmico, investigando possíveis correlações entre essas variáveis. Trinta e dois estudantes do $9^{\circ}$ ano, agrupados pela média escolar, leram dois textos diferentes, oral e silenciosamente. A velocidade calculada variou de 96,05 palavras por minuto, na leitura oral inédita, a 219,41 palavras por minuto, na segunda leitura oral. 0 texto influenciou a velocidade da leitura oral, provavelmente pela frequência ou número de caracteres das palavras. A velocidade da leitura silenciosa mostrou-se mais alta que a observada na leitura oral. Houve correlação entre velocidade de leitura e desempenho acadêmico. São necessários parâmetros brasileiros para avaliar a velocidade de leitura conforme as diferentes idades e ao níveis de escolaridade.
\end{abstract}

Palavras-chave: Adolescente; Avaliação educacional; Escolaridade; Leitura.

\begin{abstract}
Reading rate (number of words read per minute) is one of the aspects observed in reading fluency. This study characterized reading rate in the ninth grade of elementary school, according to the text and academic performance, investigating possible correlations between these variables. Thirty-two students were grouped in accordance with their average achievement scores and they read two different texts aloud and silently. Reading rate ranged from 96.05 words per minute (unpracticed reading) to 219.41 (reading aloud preceded by initial reading aloud). The text exerted an influence on reading aloud (likely the frequency and number of characters in words of the Portuguese language).

$\sim \operatorname{Pr}$

${ }^{1}$ Hospital Municipal da Criança e do Adolescente. Guarulhos, SP, Brasil

2 Universidade Federal de São Paulo, Departamento de Fonoaudiologia, Programa de Pós-Graduação em Distúrbios da Comunicação Humana. São Paulo, SP, Brasil.

3 Universidade Federal de São Paulo, Departamento de Pediatria, Curso de Especialização em Adolescência para Equipe Multidisciplinar. São Paulo, SP, Brasil.

${ }^{4}$ Universidade Federal de São Paulo, Centro de Atendimento e Apoio ao Adolescente, Serviço de Psicologia. R. Botucatu, 715, Vila Clementino, 04023-062, São Paulo, SP, Brasil. Correspondência para/Correspondence to: T.H. SCHOEN. E-mail: <teresaschoen.dped@epm.br>.
\end{abstract}


Estimated speed during silent reading was faster than the one observed during reading aloud. A correlation was found between reading rate and academic performance. Brazilian reference values are required to evaluate the reading rate at different ages and different levels of schooling.

Keywords: Adolescent; Educational measurement; Educational status; Reading.

A leitura é uma das mais importantes formas de transmissão da cultura e de integração social do ser humano (Messias, Cruz, Schallenmüller, \& Trauzettel-Klosinski, 2008). É, assim, um dos objetivos prioritários da educação fundamental. Pretende-se que crianças e jovens adquiram e melhorem progressivamente essa habilidade para, inclusive, terem acesso a novos conteúdos de aprendizagem nas diversas áreas que formam o currículo escolar (Solé, 1998).

Um importante aspecto a ser considerado para essa competência é a fluência com que a leitura se efetiva, pois o reconhecimento automático do texto permite ao leitor dedicar mais recursos cognitivos aos processos destinados à sua compreensão (Aquini, 2006; Salles \& Parente, 2002). Segundo Hudson, Lane e Pullen (2005), a leitura fluente deve conjugar três características: a acurácia (que compreende a decodificação e reconhecimento das palavras); a prosódia (que inclui a expressividade da leitura, com ritmo e entonação); e a velocidade.

À medida que o escolar se torna um leitor proficiente, passa a disponibilizar mais recursos cognitivos para a compreensão do texto e outras tarefas análogas à leitura, ou dela dependentes, como o estudo das diferentes matérias escolares, possibilitando melhor desempenho acadêmico (Pikulski \& Chard, 2005). Quanto mais rápida for a identificação de palavras, mais recursos terá a memória de trabalho para realizar outras operações (Miller \& Keenan, 2009; Perfetti, 2000).

Para que a leitura seja eficaz, fazem-se necessários, além da adequada decodificação, o domínio de regras ortográficas e gramaticais e um bom conhecimento do vocabulário. Esses elementos facilitarão o reconhecimento automático das palavras e o desenrolar da fluência leitora, com a consequente possibilidade de compreensão do texto lido

438 (Oliveira \& Santos, 2005).
Pode-se ler de forma oral ou silenciosa. $\mathrm{Na}$ leitura silenciosa, há um processo de recuperação mental da sonorização das palavras (Aquini, 2006). Nessa forma, é possível ler mais de uma palavra simultaneamente (Perfetti, 2000). É a forma de leitura mais utilizada por adultos. A leitura silenciosa é mais rápida que a leitura oral (Cavalheiro, Santos, \& Martinez, 2010; Jong, Bitler, Setten, \& Marinus, 2009; Kim, Wagner, \& Foster, 2011). Porém, é durante a leitura oral que se podem observar características como a velocidade. Esta, quando verificada por meio do número de palavras lidas por minuto, permite analisar quais rotas/estratégias de leitura o leitor utiliza (Jong et al., 2009).

O leitor competente costuma variar a rota de leitura: ou lexical, ou fonológica (Salles \& Parente, 2007). No início da alfabetização, a leitura se dá primordialmente pela rota fonológica; por isso, há uma tendência à leitura mais lenta. À medida que os escolares vão tendo maior contato com a leitura e, progressivamente, se familiarizam com número crescente de palavras, tornam-se decodificadores fluentes e leem com velocidade cada vez maior, utilizando também a rota lexical (Macedo et al., 2005; Salles \& Parente, 2002)

Ávila, Carvalho e Kida (2009) encontraram aumento progressivo da velocidade de leitura oral de acordo com a série escolar. Com o avanço da escolaridade, o aluno passa a ler mais rapidamente, pois o número de palavras lidas como um todo e armazenadas no léxico visual é maior (Cavalheiro et al., 2010; Jong et al., 2009; Macedo et al., 2005), e os processos cognitivos que intervêm são diferentes daqueles disparados durante a aprendizagem (Sternberg, 1987).

Embora se saiba que o aumento da velocidade e da acurácia devem influenciar positivamente a extração do sentido do texto, permitindo assim o bom rendimento escolar, ainda é escasso o número de informações sobre o desempenho em leitura oral 
e silenciosa por leitores brasileiros, principalmente no ciclo Il do ensino fundamental (Oliveira \& Santos, 2005). Nessa faixa de escolaridade, a maior preocupação dos educadores está voltada para a compreensão e interpretação do texto - geralmente alcançada por meio de leitura silenciosa -, e não mais para a velocidade e precisão da leitura, objetivos pertinentes aos primeiros anos do ciclo I. Dificilmente um professor de último ano do ensino fundamental solicitará a leitura oral; esse é, pois, um parâmetro ainda desconhecido. Por outro lado, quando a compreensão leitora está prejudicada, é importante que - além da investigação sobre as condições da compreensão oral, diretamente ligada à escrita (A.G.S. Capovilla, Capovilla \& Suiter, 2004) -, se conheçam também as condições de decodificação e fluência leitora, igualmente fundamentais para que o processo de compreensão ocorra livremente.

A maioria dos estudos dedica-se a identificar a velocidade e a acurácia na leitura de palavras isoladas ou pseudopalavras, e não de textos, assim como geralmente tem como foco o início da escolarização (Capellini \& Cavalheiro, 2000; Macedo et al., 2005; Nikaedo et al., 2006; Salles \& Parente, 2002; Vaessen \& Blomert, 2010). No estudo de L.S. Fuchs, Fuchs, Hosp e Jenkins (2001), a velocidade de leitura de textos explicou melhor a fluência em leitura oral que em leitura de listas de palavras. Nesse estudo, a velocidade da leitura de textos explicou uma parte substancial da variância obtida numa prova de compreensão e, portanto, esteve associada ao sucesso acadêmico. Wang, Algozzine, Ma e Porfeli (2011) afirmam ser a leitura fluente um importante fator para o desempenho acadêmico. Esses autores observaram que a velocidade de leitura aumentou com a escolaridade, e que alunos com dificuldades de aprendizagem demonstraram não só menor velocidade inicial, como também menor aumento de velocidade no decorrer do ano letivo. Assim, os autores consideraram a baixa velocidade de leitura oral como risco de problemas acadêmicos.

Assim, considerando que a velocidade de leitura pode influenciar a compreensão leitora (Corso \& Salles, 2009; Wang et al., 2011), levantou- -se a hipótese de que a baixa velocidade de leitura poderia estar correlacionada a baixos níveis de aprendizado escolar. Nesse sentido, o objetivo do presente estudo foi caracterizar a velocidade de leitura oral e silenciosa entre alunos do $9^{\circ}$ ano, segundo o texto, o tipo da leitura (inédita ou não) e o desempenho escolar.

\section{Método}

\section{Participantes}

Participaram do estudo 32 escolares (50\% do gênero masculino), todos com 14 anos (Média - $M=14,67$; Desvio-Padrão - $D P=0,31$ ), regularmente matriculados no $9^{\circ}$ ano de uma escola particular do município de São Paulo. Foram agrupados segundo as médias das matérias do $3^{\circ}$ bimestre do ano letivo em que se realizou a coleta. Um grupo foi composto por 16 escolares que apresentaram boletins com as notas mais altas no $3^{\circ}$ bimestre em todas as disciplinas (com exceção de Educação Física, Artes e Língua Estrangeira), com média entre 7,0 e $8,8(M=7,8)$. O segundo grupo foi composto pelos 16 escolares que apresentaram as notas mais baixas, com média entre 3,5 e 5,1 ( $M=4,6)$. A coleta foi realizada em outubro, após o término do $3^{\circ}$ bimestre letivo. Observou-se também uma diferença de gêneros nessa distribuição: havia mais alunos do gênero masculino (9) no grupo com notas mais baixas, e mais alunos do gênero feminino no grupo com notas mais altas (9).

Estabeleceram-se os critérios de inclusão na amostra, definidos a partir do boletim e das informações da coordenadora educacional. Todos deviam ser brasileiros natos, filhos de brasileiros natos. A língua falada em suas residências deveria ser, principalmente, o português. Todas as séries anteriores deveriam ter sido cursadas em escola brasileira, não bilíngue. Não poderiam apresentar queixas auditivas e/ou alterações visuais não corrigidas, dislexia, deficiência intelectual e/ou qualquer outra patologia que pudesse explicar diferença significativa na velocidade de leitura. Os pais deveriam assinar o Termo de Consentimento Livre e Escla- 
recido (TCLE), e os adolescentes, o Termo de Assentimento (TA).

\section{Instrumentos}

Foram selecionados dois textos diferentes, considerados equivalentes quanto ao nível de compreensão por uma professora de português do $9^{\circ}$ ano. Os textos eram desconhecidos dos participantes e foram retirados de um livro dessa série. Foram selecionados textos informativos de interesse geral e baixa dificuldade de compreensão, retirados da primeira unidade do livro. Os textos apresentavam as seguintes características: a) extensão: texto 1, com 326 palavras e 1679 caracteres; texto 2, com 373 palavras e 1693 caracteres; b) presença de palavras regulares (maioria) e irregulares, com diferentes números de sílabas; c) presença de números arábicos (365) e romanos ("século 19"). Os textos foram apresentados em fonte Arial 12 e espaçamento duplo, e impressos em folha tamanho A4, por impressora laser comercial. A escolha foi baseada nas recomendações de Deno (2003), Gonçalves (2011) e Rasinski (2006). Utilizaram-se gravador, fita cassete, cronômetro e uma ficha de leitura.

\section{Procedimentos}

O presente estudo foi submetido ao Comitê de Ética em Pesquisa da Universidade Federal de São Paulo, tendo sido aprovado sob o n 1897/06. Após o consentimento da escola participante, foram analisados os boletins escolares das quatro turmas da última série do ensino fundamental e identificados os 16 alunos com as notas mais baixas e os $16 \mathrm{com}$ as notas mais altas, como média geral do $3^{\circ}$ bimestre letivo. Os alunos foram convidados a participar, por meio de uma carta de esclarecimento sobre o estudo. Solicitou-se aos responsáveis que assinassem o TCLE, e aos alunos, o TA. Não houve recusa.

Conforme procedimento proposto por Gonçalves (2011), as leituras foram realizadas de forma individual, em uma única sessão, no horário cedido pela escola, em uma sala de aula silenciosa, com iluminação e ventilação adequadas, afastada da movimentação dos demais alunos. Os participantes permaneceram sentados em carteira escolar durante a leitura. O período de coleta durou duas semanas, correspondentes à terceira e quarta semana do mês de outubro, equivalente ao início do $4^{\circ}$ bimestre letivo. O procedimento foi realizado por dois pesquisadores simultaneamente, em salas distintas.

Os 32 escolares foram separados em dois grupos, denominados A e B, cada qual composto por oito dos alunos com as notas mais altas (média em ambos os grupos: 7,8 ) e oito com as notas mais baixas (média de todas as disciplinas, com exceção de Educação Física, Artes e Língua Estrangeira) (grupo A: média 4,6; grupo B: média 4,7).

A leitura foi dividida em duas etapas. $\mathrm{Na}$ primeira, um texto foi lido oralmente duas vezes, em que o Grupo A lia o texto 1, e o grupo B, o texto 2. Na segunda etapa, um texto foi lido primeiramente de maneira silenciosa e depois de forma oral, quando o Grupo A lia o texto 2, e o grupo B, o texto 1. Procedendo assim, cada aluno leu dois textos, duas vezes cada um, conforme estudo de Rasinski (2006), que recomenda mais de uma leitura para avaliação da fluência (Figura 1).

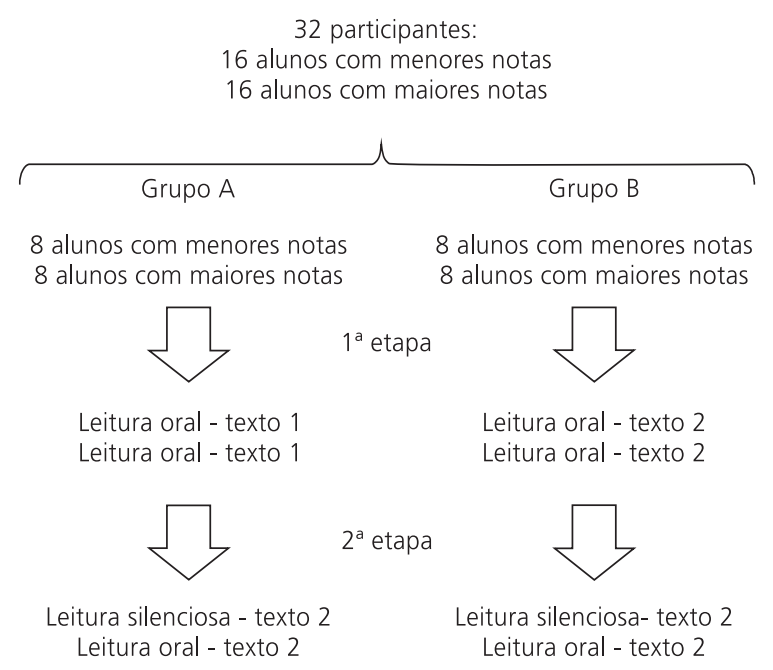

Figura 1. Tipo de leitura realizada, de acordo com o texto, por grupo, em cada etapa do estudo. São Paulo (SP), 2006. 
Os tempos das leituras orais - a primeira inédita, e a segunda já conhecida -, e das leituras silenciosas foram cronometrados e computados em segundos. A velocidade de leitura (número de palavras lidas por minuto) foi calculada pelo número total de palavras do texto, independentemente do número de sílabas ou letras (algarismos arábicos ou romanos foram considerados como uma palavra), dividido pelo tempo em segundos despendido para completar a leitura, e multiplicado por 60 . Por exemplo, se texto 1, com 326 palavras, foi lido em 181 segundos por determinado aluno, para saber a velocidade de palavras lidas por minuto, divide-se 326 palavras por 181 segundos e multiplica-se por 60 segundos, dando um total de 108,07 palavras lidas por minuto (ppm). No caso da leitura silenciosa, informava-se o momento de o aluno iniciar a leitura e ao completá-la ele dizia 'pronto', quando, então, era desligado o cronômetro. Esses valores foram reconhecidos como a velocidade de leitura estimada pelo número de palavras lidas por minuto. Não foi realizada prova de compreensão após as leituras.

Para tratamento dos dados, foram utilizados o Teste $t$ de Student Pareado, quando foram comparados os resultados entre os grupos; a Análise de Variância (Anova) (Teste F), quando se comparou a velocidade em cada texto; e a Correlação de Pearson, que mediu o quanto as variáveis notas escolares e velocidade de leitura estavam interligadas. A significância estatística adotada foi $p \leq 0,05$.

\section{Resultados}

A Tabela 1 apresenta as velocidades médias de leitura oral, observando que a segunda leitura foi significativamente mais rápida que a primeira.

Já as Tabelas 2 e 3 apresentam as velocidades médias de leitura, considerando as notas dos escolares: os 16 alunos com as notas mais baixas (oito do grupo A e oito do grupo B) (Tabela 2), e os 16 com as notas mais altas (oito do grupo $A$ e oito do grupo B) (Tabela 3). A segunda leitura oral também foi mais rápida que a primeira; e a silenciosa, mais rápida que a oral.

Comparando somente a primeira leitura, observa-se que a leitura silenciosa foi mais rápida, independentemente do texto (Teste $t$ de Student, Grupo A [texto 2] $t=-8,95 ; p<0,001$; Grupo B [texto 1] $t=-3,22 ; p=0,006$ ). As segundas leituras orais foram significativamente mais rápidas que a primeira leitura oral (Teste $t$ de Student, Grupo A

Tabela 1

Velocidade média de leitura, em palavras por minuto, por texto e por forma de leitura, em ambos os grupos, em uma escola particular do município de São Paulo no ano de 2006

\begin{tabular}{|c|c|c|c|c|c|}
\hline \multirow{2}{*}{$\begin{array}{l}\text { Grupo } A \\
N=16\end{array}$} & \multicolumn{2}{|c|}{ Texto 1 - oral } & \multicolumn{3}{|c|}{ Texto 2 silenciosa + oral } \\
\hline & $1^{\text {a }}$ leitura & $2^{a}$ leitura & $1^{\text {a }}$ leitura & & $2^{a}$ leitura \\
\hline Média & 133,78 & 148,22 & 196,14 & & 168,90 \\
\hline Desvio-padrão & 20,47 & 22,34 & 39,23 & & 24,59 \\
\hline Mínimo & 102,41 & 112,41 & 130,12 & & 130,12 \\
\hline Máximo & 171,58 & 184,53 & 294,47 & & 203,45 \\
\hline valor-p & \multicolumn{2}{|c|}{$<0,001$} & \multicolumn{3}{|c|}{0,002} \\
\hline \multirow{2}{*}{$\begin{array}{l}\text { Grupo B } \\
\mathrm{N}=16\end{array}$} & \multicolumn{2}{|c|}{ Texto 2 - oral } & \multicolumn{3}{|c|}{ Texto 1 silenciosa + oral } \\
\hline & $1^{\text {a }}$ leitura & $2^{\mathrm{a}}$ leitura & $1^{a}$ leitura & & $2^{a}$ leitura \\
\hline Média & 164,71 & 177,29 & 198,26 & & 148,48 \\
\hline Desvio-padrão & 26,18 & 30,81 & 52,02 & & 27,01 \\
\hline Máximo & 192,93 & 219,41 & 300,92 & & 181,11 \\
\hline$t$ & \multicolumn{2}{|c|}{$-2,60$} & \multicolumn{3}{|c|}{4,78} \\
\hline valor-p & \multicolumn{2}{|c|}{0,020} & \multicolumn{3}{|c|}{$<0,001$} \\
\hline
\end{tabular}


Tabela 2

Velocidade média de leitura, em palavras por minuto, por texto e por forma de leitura, dos alunos com as notas mais baixas, em ambos os grupos, em uma escola particular do município de São Paulo no ano de 2006

\begin{tabular}{|c|c|c|c|c|c|}
\hline \multirow{2}{*}{$\begin{array}{l}\text { Grupo A } \\
N=8\end{array}$} & \multicolumn{2}{|c|}{ Texto 1 - oral } & \multicolumn{3}{|c|}{ Texto 2 silenciosa + oral } \\
\hline & $1^{\text {a }}$ leitura & $2^{\mathrm{a}}$ leitura & $1^{\mathrm{a}}$ leitura & & $2^{\mathrm{a}}$ leitura \\
\hline Média & 121,60 & 136,99 & 176,80 & & 155,67 \\
\hline Desvio-padrão & 17,49 & 21,30 & 34,28 & & 25,82 \\
\hline Mínimo & 102,41 & 112,41 & 130,12 & & 130,12 \\
\hline Máximo & 147,07 & 171,58 & 223,80 & & 203,45 \\
\hline$t$ & \multicolumn{2}{|c|}{$-5,91$} & \multicolumn{3}{|c|}{2,38} \\
\hline valor-p & \multicolumn{2}{|c|}{0,001} & \multicolumn{3}{|c|}{0,049} \\
\hline \multirow{2}{*}{$\begin{array}{l}\text { Grupo B } \\
N=8\end{array}$} & \multicolumn{2}{|c|}{ Texto 2 - oral } & \multicolumn{3}{|c|}{ Texto 1 silenciosa + oral } \\
\hline & $1^{\text {a }}$ leitura & $2^{\mathrm{a}}$ leitura & $1^{a}$ leitura & & $2^{a}$ leitura \\
\hline Média & 149,55 & 156,81 & 177,76 & & 128,58 \\
\hline Desvio-padrão & 29,10 & 29,09 & 61,69 & & 24,50 \\
\hline Mínimo & 96,05 & 117,17 & 108,07 & & 90,14 \\
\hline Máximo & 179,04 & 192,93 & 300,92 & & 155,24 \\
\hline$t$ & \multicolumn{2}{|c|}{$-0,81$} & \multicolumn{3}{|c|}{2,64} \\
\hline valor-p & \multicolumn{2}{|c|}{0,446} & \multicolumn{3}{|c|}{0,033} \\
\hline
\end{tabular}

Tabela 3

Velocidade média de leitura, em palavras por minuto, por texto e por forma de leitura, dos alunos com as notas mais altas, em ambos os grupos, em uma escola particular do município de São Paulo no ano de 2006

\begin{tabular}{|c|c|c|c|c|c|}
\hline \multirow{2}{*}{$\begin{array}{l}\text { Grupo A } \\
N=8\end{array}$} & \multicolumn{2}{|c|}{ Texto 1 - oral } & \multicolumn{3}{|c|}{ Texto 2 silenciosa + oral } \\
\hline & $1^{\text {a }}$ leitura & $2^{\text {a }}$ leitura & $1^{\text {a }}$ leitura & & $2^{\text {a }}$ leitura \\
\hline Média & 145,97 & 159,45 & 215,48 & & 182,13 \\
\hline Desvio-padrão & 15,90 & 18,10 & 35,60 & & 15,13 \\
\hline Mínimo & 127,01 & 140,72 & 176,22 & & 163,36 \\
\hline Máximo & 171,58 & 184,53 & 294,47 & & 203,45 \\
\hline$t$ & \multicolumn{2}{|c|}{$-6,36$} & \multicolumn{3}{|c|}{2,80} \\
\hline valor-p & \multicolumn{2}{|c|}{$<0,001$} & \multicolumn{3}{|c|}{0,026} \\
\hline \multirow{2}{*}{$\begin{array}{l}\text { Grupo B } \\
N=8\end{array}$} & \multicolumn{2}{|c|}{ Texto 2 - oral } & \multicolumn{3}{|c|}{ Texto 1 silenciosa + oral } \\
\hline & $1^{\mathrm{a}}$ leitura & $2^{\mathrm{a}}$ leitura & $1^{\text {a }}$ leitura & & $2^{a}$ leitura \\
\hline Média & 179,87 & 197,76 & 218,75 & & 168,37 \\
\hline Desvio-padrão & 9,80 & 15,16 & 32,15 & & 7,64 \\
\hline Mínimo & 163,36 & 177,62 & 171,58 & & 159,02 \\
\hline Máximo & 192,93 & 219,41 & 271,67 & & 181,11 \\
\hline$t$ & \multicolumn{2}{|c|}{$-5,38$} & \multicolumn{3}{|c|}{4,65} \\
\hline valor-p & \multicolumn{2}{|c|}{0,001} & \multicolumn{3}{|c|}{0,002} \\
\hline
\end{tabular}

[texto 1] $t=-9,35 ; p<0,001 ;$ Grupo B [texto 2] 준 $t=13,65 ; p<0,001)$, e sua velocidade dependeu do texto (Teste $\mathrm{F}$ texto $1-5,002 ; p=0,033$; texto 2 - 9,331; $p=0,005)$. A velocidade da leitura oral inédita também dependeu do texto (Teste $F$ 13,86; $p=0,001)$. Em relação à leitura silenciosa, não houve interferência do texto (Teste $\mathrm{F} 0,01 ; p=0,897$ ).

442 Observando-se as Tabelas 2 e 3, verifica-se que o aumento na velocidade na segunda leitura oral, com leitura oral prévia, independeu do desempenho acadêmico, embora os alunos com as notas mais altas tenham aumentado a velocidade, independentemente do texto (Teste $t$ de Student: alunos com notas mais baixas no Grupo A, $t=-7,56$ e $p<0,001$; notas mais baixas no Grupo $B, t=-1,48$ e $p=0,183$; alunos com notas mais altas no Grupo $A, t=-5,87$ 
Tabela 4

Correlação entre a velocidade de leitura e as notas nas disciplinas em uma escola particular do município de São Paulo no ano de 2006

\begin{tabular}{|c|c|c|c|c|c|}
\hline \multirow{2}{*}{ Disciplinas } & & \multicolumn{2}{|c|}{ Etapa I } & \multicolumn{2}{|c|}{ Etapa II } \\
\hline & & $1^{\mathrm{a}}$ leitura oral & $2^{\mathrm{a}}$ leitura oral & $1^{a}$ leitura oral & $2^{a}$ leitura oral \\
\hline \multirow[t]{2}{*}{ Português } & $r$ & 0,45 & 0,41 & 0,45 & 0,54 \\
\hline & valor-p & $0,010^{*}$ & $0,021^{*}$ & $0,009^{*}$ & $0,001^{*}$ \\
\hline \multirow[t]{2}{*}{ Matemática } & $r$ & 0,38 & 0,40 & 0,46 & 0,55 \\
\hline & valor-p & $0,030^{*}$ & $0,022^{*}$ & $0,008^{*}$ & $0,001^{*}$ \\
\hline \multirow[t]{2}{*}{ História } & $r$ & 0,53 & 0,52 & 0,45 & 0,48 \\
\hline & valor- $p$ & $0,002^{*}$ & $0,002^{*}$ & $0,010^{*}$ & $0,006^{*}$ \\
\hline \multirow[t]{2}{*}{ Geografia } & $r$ & 0,35 & 0,35 & 0,34 & 0,45 \\
\hline & valor-p & $0,047^{*}$ & $0,050^{*}$ & $0,054^{*}$ & $0,009^{*}$ \\
\hline \multirow[t]{2}{*}{ Ciências } & $r$ & 0,40 & 0,44 & 0,46 & 0,55 \\
\hline & valor-p & $0,024^{*}$ & $0,011^{*}$ & $0,009^{*}$ & $0,001^{*}$ \\
\hline \multirow[t]{2}{*}{ Média } & $r$ & 0,43 & 0,44 & 0,46 & 0,54 \\
\hline & valor-p & $0,013^{*}$ & $0,012^{*}$ & $0,008^{*}$ & $0,001^{*}$ \\
\hline \multirow[t]{2}{*}{ Média sem Matemática } & $r$ & 0,45 & 0,45 & 0,45 & 0,54 \\
\hline & valor-p & $0,010^{*}$ & $0,010^{*}$ & $0,009^{*}$ & $0,001^{*}$ \\
\hline
\end{tabular}

Nota: ${ }^{*} p \leq 0,05$.

e $p-0,001$; notas mais altas no Grupo $B, t=-4,06$ e $p=0,005)$.

A Tabela 4 mostra que todas as variáveis de rendimento escolar (notas em cada disciplina ou média) correlacionaram-se positivamente, em uma magnitude moderada, com as velocidades de leitura em todos os procedimentos utilizados para avaliação.

\section{Discussão}

Este estudo buscou avaliar a velocidade de leitura de escolares da última série do ensino fundamental, por meio de textos. Duas são as diferenças desta pesquisa em relação a outros estudos brasileiros: a amostra, que teve como foco adolescentes; e o instrumento, constituído por textos, e não por palavras ou sentenças. A literatura recomenda que a velocidade de leitura seja avaliada em textos contínuos e com várias linhas (Deno, 2003; Gonçalves, 2011; Messias et al., 2008). Para este estudo não foi encontrado nenhum texto ou teste padronizado, razão pela qual foram utilizados textos retirados de livros escolares (Deno, 2003; Rasinski, 2006).

Os Grupos A e B, com estudantes dos dois níveis de desempenho acadêmico, mostraram, nas primeiras leituras orais, a média de 133,78 ppm no primeiro texto, e de 164,71 ppm no segundo. Considerando as informações encontradas na literatura (Ávila et al., 2009; Whittaker \& Lovie-Kitchin, 1993), essas seriam médias adequadas, fluentes e rápidas. Messias et al. (2008) encontraram médias mais altas em adultos com ensino médio. Em relação ao trabalho de Castro, Kallie e Salomão (2005), os adolescentes do $9^{\circ}$ ano deste estudo mostraram desempenho esperado, com média de velocidade inferior aos adultos e superior aos escolares da $3^{a}$ série.

Na Etapa I do presente estudo, quando as leituras foram realizadas oralmente, a segunda leitura foi mais rápida em ambos os grupos, independentemente do texto apresentado. De fato, a leitura já fluente implica algum nível de compreensão e, dessa forma, deve promover maior acurácia e aumento da velocidade em uma segunda leitura do mesmo texto. Os resultados encontrados também podem indicar que algumas palavras, inicialmente lidas pela rota fonológica, passaram a serem lidas pela rota lexical (Macedo et al., 2005). Também pode indicar que os alunos utilizaram de forma adequada sua memória de trabalho; por exemplo, tendo antes hesitado na leitura de alguma palavra, não titubearam quando esta se apresentou novamente, o que indica que melhoraram o desempenho 
com o treino (Solé, 1998). Esse resultado foi mais bem observado quando se analisou o total de escolares de ambos os grupos.

Entretanto, o subgrupo B com notas mais baixas não apresentou o mesmo padrão de desempenho. Pesquisas relatam que valores mais baixos de velocidade de leitura podem ser determinados pela presença de maior quantidade de erros durante essa atividade (Kim, Davis, Burnham, \& Luksaneeyanawin, 2004; Talcott et al., 2003). Embora a acurácia não tenha sido abordada no presente estudo, provavelmente possa ter influenciado o resultado. Em alguns trabalhos, já se havia observado que escolares com problemas de aprendizagem leem mais lentamente, tanto oral quanto silenciosamente (Capellini \& Cavalheiro, 2000). Os escolares com dificuldades de leitura podem apresentar déficits de fluência leitora e limitações para compreender o texto lido, em decorrência de alterações do processamento da informação fonológica e da baixa capacidade de armazenamento na memória de trabalho (Van der Leij \& Morfidi, 2006). Autores sugerem que as dificuldades de maus leitores, em comparação com os bons leitores, estejam relacionadas principalmente ao processamento fonológico, que inclui a consciência fonológica, o vocabulário receptivo auditivo e a memória fonológica, habilidades que envolvem processamento da informação baseada na estrutura fonológica da linguagem oral (Capovilla et al., 2004). De qualquer forma, os resultados da segunda leitura do Grupo B de notas mais baixas mostram que nem sempre o treino da leitura resulta em melhora significativa da fluência; em alguns casos, nota-se a necessidade de investigação e suporte terapêutico.

Tanto a primeira quanto a segunda leitura do texto 1 mostraram menores valores médios de número de palavras lidas por minuto, quando comparados com as leituras do texto 2. Messias et al. (2008) e Castro et al. (2005) também encontraram diferença estatisticamente significante entre as velocidades de leitura de acordo com o texto. Esses resultados sugerem que características dos textos podem influenciar a velocidade de reconhecimento das palavras. O padrão recorrente, observado como aumento do número de palavra lidas por minuto à segunda leitura, mostra que, apesar de os participantes estarem no último ano do ensino fundamental, a prática da leitura ainda pode interferir na velocidade.

Este trabalho não se deteve em analisar a leitura de palavras específicas ou isoladas, embora se tenha percebido uma dificuldade dos escolares com o algarismo romano contido no texto ("...no final do século XIX"). Quase todos hesitaram nesse reconhecimento, o que pode ter sido o motivo de terem lido o texto 2 com velocidade mais baixa que o texto 1. Todavia, esse item não foi considerado impeditivo, visto ser conteúdo de $4^{\circ}$ ano escolar, e utilizado em textos didáticos de História. O êxito do reconhecimento da palavra depende muito das suas características. Cada texto é particular, pois pode variar em número de palavras, número de sílabas de cada palavra, regularidade ortográfica, frequência das palavras na língua portuguesa (oral ou escrita), frequência das palavras no texto, o fato de serem ou não palavras derivadas, além da dificuldade do assunto, que pode ser conhecido ou não. Por outro lado, velocidades mais baixas de leitura podem estar associadas a maior quantidade de erros durante essa atividade (Kim et al., 2004), mas esse aspecto não foi abordado no presente estudo.

A leitura silenciosa foi mais rápida que a leitura oral e não teve influência do texto, como encontrado no estudo de Cavalheiro et al. (2010). Diferentemente, Capellini e Cavalheiro (2000) observaram que o texto também tem poder na leitura silenciosa; entretanto, esses autores descreveram escolares de $2^{a}$ a $4^{a}$ série, quando a leitura silenciosa talvez ainda utilize muitos processos de reconhecimento fonológico das palavras, visto ainda estarem as crianças em processo de alfabetização. Já o avanço da escolaridade e a familiaridade com textos escritos permitem que o adolescente utilize outras rotas de leitura. Sternberg (1987) afirma que os processos cognitivos que intervêm no que já foi aprendido são diferentes dos que intervêm na aprendizagem de uma habilidade.

O modo silencioso permite a leitura de mais de uma palavra ao mesmo tempo (Perfetti, 2000), de forma que o leitor não precisa fixá-las isola- 
damente, mas pode obter as informações enquanto o olho está se movendo, ou trabalhar com unidades de processamento na memória de trabalho durante a leitura do texto. Na leitura oral todos os fonemas são pronunciados, enquanto na silenciosa basta sua identificação. Um exemplo que torna a leitura silenciosa mais rápida pode ser a leitura de números; "1985", visto como uma única unidade na leitura silenciosa, será lido oralmente como mil, novecentos e oitenta e cinco. Da mesma forma, expressões comuns, como "de repente" ou "a respeito" são lidas silenciosamente também como uma única unidade.

Diversos estudos mostram a relação entre velocidade de leitura e escolaridade, uma vez que alunos das séries ou classes mais adiantadas apresentam leitura mais fluente (Mather \& Goldstein, 2001; Stivanin \& Scheuer, 2007; Wang et al., 2011). Capellini e Cavalheiro (2000) observaram que crianças com problemas de aprendizagem leem mais lentamente. Os resultados do presente trabalho vão ao encontro desses estudos, pois mostraram que os alunos com notas mais baixas apresentaram valores mais baixos de velocidade de leitura. A leitura fluente é decorrente da escolarização - séries mais avançadas devem apresentar melhor fluência leitora -, e o leitor fluente costuma ter mais facilidade no aprendizado. Aqueles que leem fluentemente, com boa velocidade e baixo número de erros, têm maior probabilidade de compreender o que estão lendo (Aquini, 2006; Ávila et al., 2009; Luís, 2010).

Aventa-se aqui a possibilidade de os bons alunos apresentarem maior vocabulário, ou maior velocidade para decodificar, o que facilita a leitura pela rota lexical. A rota lexical acessa e recupera de forma direta o significado da palavra reconhecida, sendo usada para a leitura de uma palavra previamente armazenada no léxico (Stivanin \& Scheuer, 2007). Outro tipo de rota utilizada na leitura é a fonológica, que envolve a conversão grafema-fonema, geralmente utilizada para a leitura de palavras não estocadas na memória. Palavras compostas de sílabas regulares são facilmente lidas por essa rota, que é mais utilizada no início da alfabetização ou em textos com vocabulário inédito.
Espera-se que os alunos da última série do ensino fundamental tenham vocabulário de palavras escritas suficientemente abrangente para dar conta dos diversos textos informativos utilizados no cotidiano acadêmico. A despeito da hipótese de que alunos mais fracos academicamente apresentassem maior dificuldade em leitura, os alunos que participaram deste estudo não eram disléxicos ou portadores de qualquer outra patologia que pudesse explicar diferença significativa na velocidade de leitura. Aventa-se, então, a possibilidade de que uma intervenção focada na leitura pudesse beneficiar o rendimento em todas as disciplinas escolares. O aprimoramento da leitura deve ser preocupação permanente de todos os professores, independentemente da disciplina que ministram (Silva, 2004; Solé, 1998), pois, como visto, a velocidade de leitura esteve associada a todas as matérias, inclusive Matemática. Embora a fluência, isoladamente, não seja garantia de sucesso acadêmico, ela libera o indivíduo para focar sua atenção em outras questões, especialmente na compreensão do material lido. É preciso saber utilizar diferentes fontes de informação e recursos tecnológicos para adquirir conhecimento e trabalhar com ele. A maioria das fontes utiliza a linguagem escrita; portanto, é fundamental que a leitura seja fluente para facilitar a inclusão na sociedade contemporânea (Messias et al., 2008). Henriques e Soares (2008) acreditam que os alunos precisam desenvolver habilidades que lhes permitam integrar-se ao mundo de tecnologias e mudanças diárias, e a destreza na leitura é uma delas.

Este estudo apresenta algumas limitações, uma vez que o tamanho e a seleção da amostra, restrita a uma escola da rede particular, limitam a generalização dos resultados encontrados. Além disso, pode-se considerar que a leitura oral mostrou-se mais rápida na segunda leitura não inédita, em todas as condições de agrupamento dos escolares, com exceção de um subgrupo de notas mais baixas. Para entender esses resultados, são necessários estudos longitudinais ou avaliações clínicas para investigar, sob ambos os pontos de vista, a origem dessa diferença de padrão de desempenho (Kim et al., 2011). 
Os resultados obtidos neste estudo devem ser considerados como uma tentativa inicial de verificar as velocidades de leitura de escolares adolescentes, visto estarem restritos a uma única série e uma única escola. Os alunos de $9^{a}$ série leram dois textos diferentes, em duas formas diferentes (oral e silenciosa). O número de palavras lidas por minuto variou de acordo com o texto; com a forma, sendo a leitura silenciosa mais rápida que a oral; e com o desempenho acadêmico, sendo que os alunos com maiores notas leram mais palavras por minuto. A segunda leitura oral foi mais rápida que a primeira leitura oral, mostrando a importância do treino nesse tipo de tarefa.

Este estudo ressalta a necessidade de se fixarem parâmetros para avaliar a velocidade de leitura - um dos componentes da fluência -, no português falado no Brasil, em textos padronizados e de acordo com a série escolar. Outros estudos são necessários, também em escolas públicas, para melhor verificação da influência da velocidade de leitura no desempenho acadêmico, de preferência longitudinal, como ocorrido no estudo de Wang et al. (2011) e verificando os tipos de erros cometidos pelos leitores.

\section{Referências}

Aquini, J. M. P. M. (2006). A leitura oral expressiva como variável facilitadora da compreensão (Dissertação de mestrado não-publicada). Pontifícia Universidade Católica do Rio Grande do Sul, Porto Alegre.

Ávila, C. R. B., Carvalho, C. A. F., \& Kida, A. S. B. (2009). Parâmetros de fluência e compreensão de leitura. In T. Barbosa, C. C. Rodrigues, C. B. Mello, S. A. Capellini, R. Mousinho, \& L. M. Alves (Orgs.), Temas em dislexia. São Paulo: Artes Médicas.

Capellini, A. S., \& Cavalheiro, L. G. (2000). Avaliação do nível e velocidade de leitura em escolares com e sem dificuldade na leitura. Temas sobre Desenvolvimento, 9(51), 5-12.

Capovilla, A. G. S., Capovilla, F. C., \& Suiter, I. (2004). Processamento cognitivo em crianças com e sem dificuldade de leitura. Psicologia em Estudo, 9(3), 449-58.

Castro, C. T. M., Kallie, C. S., \& Salomão, S. R. (2005). Elaboração e validação de tabela MNREAD para o idioma português. Arquivos Brasileiros de Oftalmologia, 68(6), 777-783.
Cavalheiro, L. G., Santos, M. S., \& Martinez, P. C. (2010). Influência da consciência fonológica na aquisição de leitura. Revista CEFAC, 12(6), 1009-1016.

Corso, V. H., \& Salles, J. (2009). Relação entre leitura de palavras isoladas e compreensão de leitura textual em crianças. Letras Hoje, 44(3), 28-35.

Deno, S. L. (2003). Developments in curriculum-based measurement. The Journal of Special Education, 37(3), 184-192.

Fuchs, L. S., Fuchs, D., Hosp, M. K., \& Jenkins, J. R. (2001). Oral reading fluency as an indicator of reading competence: A theoretical, empirical, and historical analysis. Scientific studies of Reading, 5(3), 239-256.

Gonçalves, D. (2011). Avaliação da fluência da leitura oral e dificuldades na aprendizagem: aplicações clínicas e educacionais. In VIII Congresso Anual Iberoamericano de Avaliação Psicológica. Faculdade de Psicologia da Universidade de Lisboa, Lisboa.

Henriques, V. F. C. S., \& Soares, A. B. (2008). A compreensão de textos em condições de compartilhamento da atenção. Psicologia em Pesquisa, 2(2), 87-95.

Hudson, R., Lane, H., \& Pullen, P. (2005). Reading fluency assessment and instruction: What, why, and how? The Reading Teacher, 58(8), 702-714.

Jong, P. F., Bitler, D. J. L., Setten, M., \& Marinus, E. (2009). Does phonological recoding occur during silente reading, and is it necessary for orthographic learning? Journal of Experimental Child Psychology, 104(3), 267-282.

Kim, J., Davis, C., Burnham, D., \& Luksaneeyanawin, S. (2004). The effect of script on poor readers' sensitivity to dynamic visual stimuli. Brain and Language, 91(3), 326-335.

Kim, Y. S., Wagner, R., \& Foster, E. (2011). Relations among oral Reading fluency, silente reading fluency, and reading comprehension: A latente variable study of first-grade readers. Scientific Studies of Reading, 15(4), 338-362.

Luís, J. (2010). Leitura oral em alunos do $4^{\circ}$ ano do $1^{\circ}$ ciclo: avaliação da velocidade, correcção e compreensão (Tese de mestrado não-publicada). Universidade de Lisboa. Recuperado em maio 4, 2014, de http://hdl.handle.net/10451/1907

Macedo, E. C., Capovilla, F. C., Nikaedo, C. C., Orsati, F. T., Lukasova, K., Capovilla, A. G. S., \& Diana, C. (2005). Teleavaliação da habilidade de leitura no ensino infantil fundamental. Psicologia Escolar e Educacional, 9(1), 37-46.

Mather, N., \& Goldstein, S. (2001). Learning disabilities and challenging behaviors: A guide to intervention and classroom management. Baltimore: Brookes.

Messias, A., Cruz, A. A. V., Schallenmuller, S. J., \& Trauzettel-Klosinski, S. (2008). Textos padronizados em português (BR) para medida da velocidade de leitura: comparação com quatro idiomas europeus. Arquivos Brasileiros de Oftalmologia, 71(4), 553-558. 
Miller, A. C., \& Keenan, J. M. (2009). How word decoding skill impacts text memory: The centrality deficit and how domain knowledge can compensate. Annals of Dyslexia, 59(2), 99-113.

Nikaedo, C. C., Macedo, E. C., Diana, C., Kukasova, K., Kuriyama, C., Orsati, F., ... Natalle, L. (2006). Nível de leitura e compreensão de sentenças faladas no ensino fundamental: diagnóstico diferencial dos problemas de leitura. Psicopedagogia, 23(71), 107-115.

Oliveira, K. L., \& Santos, A. A. A. (2005). Compreensão em leitura e avaliação da aprendizagem em Universitários. Psicologia: Reflexão e Crítica, 18(1), 118-124.

Perfetti, C. A. (2000). A capacidade para leitura. In R. J. Sternberg. As capacidades intelectuais humanas. Porto Alegre: Artes Médicas.

Pikulski, J. J., \& Chard, D. J. (2005). Fluency: Bridge between decoding and reading comprehension. The Reading Teacher, 58(6), 510-519.

Rasinski, T. (2006). Reading fluency instruction: Moving beyong accuracy, automaticity, and prosody. The Reading Teacher, 59(7), 704-706.

Salles, J. F., \& Parente, M. A. M. P. (2002). Processos cognitivos na leitura de palavras em crianças: relações com compreensão e tempo de leitura. Psicologia: Reflexão e Crítica, 15(2), 321-331.

Salles, J. F., \& Parente, M. A. M. P. (2007). Avaliação da leitura e escrita de palavras em crianças de $2^{a}$ série: abordagem neuropsicológica cognitiva. Psicologia: Reflexão e Crítica, 20(2), 220-228.
Silva, V. R. (2004). Estratégias de leitura e competência leitora: contribuições para a prática de ensino em História. História, 23(1-2), 69-83.

Solé, I. (1998). Estratégias de leitura. Porto Alegre: ArtMed.

Sternberg, R. J. (1987). Inteligencia humana: la naturaleza de la inteligencia y su medición. Barcelona: Paidós.

Stivanin, L., \& Scheuer, C. I. (2007). Tempo de latência para a leitura: influência da freqüência da palavra escrita e da escolarização. Revista da Sociedade Brasileira de Fonoaudiologia, 12(3), 306-213.

Talcott, J. B., Gram, A., Ingelghem, M., Witton, C., Stein, J. F., \& Toennessen, F. E. (2003). Impaired sensitivity to dynamic stimuli in poor readers of a regular orthography. Brain and Language, 87(2), 259-66.

Vaessen, A., \& Blomert, L. (2010). Long-term cognitive dynamics of fluent reading development. Journal of Experimental Child Psychology, 105(3), 213-231.

Van der Leij, A., \& Morfidi, E. (2006). Core deficits and variable differences in Dutch poor readers learning English. Journal Learning Disabilities, 39(1),74-90.

Wang, C., Algozzine, B., Ma, W., \& Porfeli, E. (2011). Oral reading rates of second-grade students. Journal of Educational Psychology, 103(2), 442-454.

Whittaker, S. G., \& Lovie-Kitchin, J. (1993). Visual requirements for reading. Optometry and Vision Science, 70(1), 54-65.

Recebido: novembro 14, 2012

Versão final: maio 7, 2014

Aprovado: julho 11, 2014 
